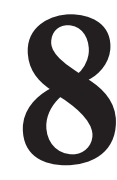

\title{
LA UNIVERSIDAD POSTMODERNA Y LA NUEVA CREACIÓN DEL CONOCIMIENTO
}

\author{
(THE POSTMODERN UNIVERSITY AND THE NEW KNOWLEDGE \\ PRODUCTION)
}

María José García Ruiz

UNED

\section{RESUMEN}

La globalización y su potenciación de la dimensión económica de las sociedades occidentales han favorecido la ascendencia y el influjo de la visión postmoderna de la universidad y del paradigma de la nueva creación del conocimiento por dicha visión propuesto. Los dos modos de creación del conocimiento, Modo 1 y Modo 2, constituyen dos paradigmas opuestos que concretan epistemológicamente los dos modelos, moderno y postmoderno, de universidad que coexisten sin relacionarse en los primeros años del siglo XXI. La masificación de la universidad constituye el argumento más potente de los defensores de la nueva epistemología. Si bien no se puede negar la existencia y el crecimiento de los nuevos desarrollos epistemológicos, quedan aspectos no resueltos del mismo necesitados aún de mucho debate académico. Entre estos destacan dos: la nueva composición de las identidades profesionales, y el presumible déficit democrático del Modo 2 de producción del conocimiento.

\section{ABSTRACT}

Globalisation, and its promotion of the Economic dimension of Western societies, has promoted the upward and influence of the Postmodern vision of the university and of the paradigm of the new knowledge production proposed by such vision. The two modes of knowledge production, Mode 1 and Mode 2, constitute two conflicting paradigms that realize epistemologically the 
two models of university, Modern and Postmodern, that coexist without relation in the first years of the XXIst century. The massification of the university constitutes the strongest argument of the advocates of the new epistemology. Although the existence and the increase of the new epistemological developments cannot be denied, there remain certain aspects not yet fully resolved of the new knowledge production paradigm which yet need further academic debate. Among such aspects two can be highlighted: the new composition of professional identities, and the presumed democratic deficit of Mode 2 knowledge production.

\section{INTRODUCCIÓN}

En los círculos académicos, ha habido y sigue habiendo mucho debate sobre la naturaleza y el significado de la globalización. Hay autores que afirman que la globalización "constituye una nueva y específica forma de relación entre los Estados- nación y la economía mundial»(Dale, 2007, 48) por la cual los «gobiernos nacionales son constreñidos a buscar la modernización de sus economías nacionales (...) y a potenciar la capacidad de competición internacional de sus sistemas de bienestar nacionales» (Dale, 2007, 49). Sea cual sea la definición que se suscriba sobre la globalización y las coordenadas geográfico-temporales en las que se ubique su génesis, no parece haber acuerdo sobre si el proceso de globalización ejerce un impacto unificador y homogéneo en los diversos países sometidos a su influjo o si, por el contrario, sus efectos tienden a la diversificación de las políticas nacionales. Hay autores que se refieren a esta ambivalencia como a la «paradoja» de la globalización (Dale, 2007). Quizá las realidades de «unificación» y «homogeneización» sean las que, de forma más inmediata, se asocien al proceso de globalización cuando éste es evocado. Y ello no es extraño si se tiene en cuenta que, una de las respuestas colectivas de los Estados-nación al proceso de globalización, ha radicado en el establecimiento de una serie de organizaciones internacionales (la OCDE, el Banco Mundial, la Unión Europea, etc.), a las que los diversos países han cedido voluntariamente un porcentaje de su potestad política nacional en aras a mantener sus propias posiciones privilegiadas en la economía mundial. Este influjo unificador es también patente en tanto que dichas organizaciones se rigen por una ideología común con rasgos clave, de índole económica, que constituyen los «filtros ideológicos», que informan la dirección a adoptar por las decisiones de la política nacional. La globalización, en este sentido, crea retos similares para los Estados, los cuales emiten sus posibles respuestas en modos similares (Dale, 2007).

Pero, por otra parte, hay autores que realizan un énfasis preferente en el potencial diversificador de la globalización (Dale, 2007; Gibbons, 2004). 
El argumento central de estos autores radica en que, frente a los tradicionales mecanismos de influjo político externo (préstamo de políticas, borrowing policies, aprendizaje de políticas, learning policies), los mecanismos de influjo político externo de la globalización poseen unas características, cualitativamente diversas de los anteriores, que suponen una fuente de diversificación en las políticas nacionales sometidas a su influjo (Dale, 2007). Según estos autores, los mismos nuevos mecanismos a través de los cuales se opera la globalización (armonización, diseminación, estandarización, establecimiento de interdependencia, imposición) (Dale, 2007), no son meros conductos neutros, sino que ellos mismos condicionan y modifican la naturaleza del efecto que suscitan, especialmente en las tres formas principales de regionalización (Europa, Asia y América). Sin entrar a detallar la especificidad de cada mecanismo de globalización, decir que las características de los mismos frente a los mecanismos tradicionales de influjo político externo se cifran en que su ámbito de acción no sólo abarca los procesos políticos, sino que incluye los objetivos políticos; que son iniciados de forma externa, y que recurren a un amplio espectro de formas de poder. Según esta postura, la globalización no sólo no ha reducido a obsoletos a los Estados-nación, sino que los rasgos sociales y culturales, así como el espectro de políticas nacionales específicas existentes, constituyen estructuras a las cuales se deben adaptar las innovaciones (Dale, 2007), garantizándose con ello la pervivencia de la diversidad.

El fenómeno de la globalización ejerce sus efectos unificadores y diversificadores en la realidad de los paradigmas educativos que se desarrollan en los sistemas educativos contemporáneos. Este fenómeno y la expansión sin precedentes de las nuevas tecnologías han generado, a comienzos del siglo XXI, un contexto propicio para el cuestionamiento de la validez de los paradigmas educativos tradicionales y la propuesta de cambios paradigmáticos más acordes con la realidad actual de las sociedades occidentales. Este espíritu de transformación está siendo particularmente promovido por los académicos que se autodenominan postmodernos, a través de su innovadora y, en la mayoría de los casos, rupturista, visión de los diversos aspectos sociales y educativos de la sociedad contemporánea.

Un paradigma está compuesto por «las creencias, valores, teorías, modelos y técnicas empleados por los investigadores para legitimar su trabajo o direccionar sus investigaciones» (Holmes, 1986, 180). Como nos advierte Holmes, las teorías en el ámbito de las Ciencias Sociales nunca alcanzan el estatus de aquellas que se ubican en el entorno de las Ciencias Naturales. De suerte que, cuando se producen cambios paradigmáticos en las Ciencias Sociales, estos cambios no sólo son solamente aceptados por un reducido grupo de pensadores radicales, sino que, de hecho, las teorías y creencias que preceden las revoluciones científicas, continúan informan- 
do el trabajo de los investigadores. Así, hay académicos que basan su trabajo en los paradigmas creados por Platón, y otros investigadores sociales trabajan con paradigmas alternativos. Los cambios paradigmáticos en Ciencias Sociales rara vez implican el rechazo total de un paradigma existente. En las Ciencias Sociales lo que se produce, según la acertada visión de Holmes, es la coexistencia de paradigmas y la existencia de paradigmas en competición. Estos paradigmas pueden compartir algunos presupuestos y teorías, y diferir en otros.

En las líneas que siguen vamos a analizar un aspecto educativo que, en la actualidad, está experimentando un cambio de paradigma: la naturaleza de la epistemología contemporánea o el carácter de la creación del conocimiento. En relación a este paradigma, hay una clara polarización de posturas entre académicos tradicionales y progresistas, o entre académicos modernos y postmodernos.

\section{NUEVA CONCEPCIÓN DEL CONOCIMIENTO}

En la era de la globalización, la institución universitaria constituye una de las entidades que está siendo sometida a mayor escrutinio político y social. También están siendo sometidos a intenso análisis el conjunto de procesos que tienen lugar dentro del quehacer universitario y, en concreto, la naturaleza del proceso de la creación del conocimiento. Los académicos postmodernos son los autores que defienden en mayor medida la radicalidad del cambio en la universidad y en dinámicas como la creación del conocimiento, transformación que es cuestionada por los académicos modernistas.

Académicos como Gibbons, Limoges, Nowotny, Schwartzman, Scott y Trow han cifrado la masificación de la enseñanza y la investigación como el factor crucial de las actuales transformaciones que se están produciendo en el ámbito universitario y en sus procesos. Entre los cambios que se han producido en la universidad debidos al factor de la masificación de la enseñanza y la investigación destacan aquellos habidos en el modo de producción del conocimiento (Gibbons et al., 1994).

Hay una serie de tendencias epistemológicas que advierten de la existencia de cambios en el modo de producción del conocimiento en la sociedad contemporánea. Junto al modo disciplinar tradicional de producción del conocimiento (Modo 1) está surgiendo el Modo 2, creado en contextos transdisciplinares sociales, políticos y económicos más amplios. El Modo 1 engloba a un conjunto de ideas, métodos, valores y normas cognitivas y sociales que ratifican la bondad del modelo newtoniano de producción del conocimiento y legitiman el carácter y la naturaleza del desarrollo episte- 
mológico de las instituciones. El Modo 2 se configura a partir de una serie de atributos coherentes y estables que atestiguan la plausibilidad de un modelo heterogéneo y socialmente distribuido de creación del conocimiento, en el que aún permanecen sin definir ciertos parámetros relativos a la naturaleza epistemológica del conocimiento, como la entidad de la calidad del conocimiento.

La causa del surgimiento del conocimiento Modo 2 reside en la masificación de la enseñanza superior iniciada en los países occidentales en la segunda postguerra. El número de egresados de las instituciones de enseñanza superior con altas competencias investigadoras ha sido demasiado elevado para poder ser absorbido por estas instituciones. Dichos egresados han desarrollado su labor investigadora en un gran número de instituciones sociales alternativas, provocando la emergencia de un gran contingente de instituciones sociales que compiten con las universidades en su función de creación de conocimiento.

El Modo 1 y el Modo 2 de producción del conocimiento poseen rasgos constitutivos esencialmente diversos. Así, el Modo 1 de producción de conocimiento se genera en un contexto disciplinar regido por los intereses académicos de una comunidad específica, y posee las características de la homogeneidad y la jerarquía. Frente a estos atributos, el Modo 2 se caracteriza por su emergencia en un contexto de aplicación, heterogeneidad, ausencia de jerarquía, transitoriedad y responsabilidad social.

La aparente creciente ascendencia del Modo 2 de producción del conocimiento supone unas implicaciones respecto a la academia tradicional que merecen una consideración detenida. La primera de estas implicaciones alcanza al ethos organizativo, funcional y teleológico de las instituciones de enseñanza superior. Los proponentes y defensores del Modo 2 de producción del conocimiento abogan por el replanteamiento funcional y teleológico de las tradicionales universidades. Estos proponentes elogian las instituciones de enseñanza superior que ya funcionan desde los nuevos parámetros del cambio, hecho visible en el tipo de personal que contratan y en los acuerdos específicos de colaboración que ratifican. Así, una institución paradigmática que gozaría del favor de los partidarios del Modo 2 de producción del conocimiento y de sus exigencias de innovación sería la Universidad para la industria británica. Y, por lo mismo, una institución que estaría en las antípodas de la permeabilidad exigida por estos autores a las instituciones académicas en el siglo XXI sería la Universidad de Oxford. El carácter emblemático de estas dos instituciones de ethos tan contrapuestos ejemplariza de forma gráfica la polaridad de discursos moderno versus postmoderno que actualmente se debaten en el mundo académico. Sin que aboguemos por una postura cerrada e intransigente de defensa a ultranza de la tradición 
académica universitaria, parece inevitable que el potencial predominio del Modo 2 en el mundo académico occidental supondría la victoria del modelo postmoderno de la enseñanza superior y, más ampliamente, de dicho modelo en su comprensión del mundo y del hombre. Este modelo posee, a nuestro parecer, incógnitas e implicaciones de corte relativista, presentista y transitorio que distan de poseer la solidez argumental y la garantía existencial que ofrece la tradicional doctrina modernista.

Una segunda implicación que se colige del Modo 2 de producción del conocimiento apunta a su mayor afinidad por las ciencias empíricas y aplicadas (ingenierías química y aeronáutica, ciencia de los ordenadores, etc.) que por otras como las humanidades, a las cuales exige una remodelación en la línea de una mayor responsividad social. Así, y en este mismo sentido, «la naturaleza de los problemas abordados en el Modo 2 es cambiante y transitoria» (Gibbons et al., 1997, 18). Es decir, una doctrina y una práctica contrariamente diversa a las prácticas epistemológicas de las humanidades, las cuales reinterpretan de forma cíclica y constante la misma entidad de problemáticas humanas a la luz del contexto de los nuevos tiempos. Es discutible, desde nuestro punto de vista, que las humanidades deban modificar su ethos investigador en aras a responder en mejor medida a las exigencias de los presupuestos postmodernos. Pues estas ciencias deben su tributo a las profundas y recurrentes preocupaciones humanísticas, mucho antes que a una corriente sociohistórica con la que muchos académicos afirman no reconocer ni comulgar con sus presupuestos, como es el caso del académico Filmer (Filmer, 1997). Junto a esta implicación que se deduce de los presupuestos del Modo 2 de producción del conocimiento, hay otro imperativo epistemológico del Modo 2 que violenta la misión y las prácticas académicas tradicionalmente imperantes en la universidad. Se trata del atributo de responsabilidad social que impregna el proceso de producción del conocimiento en el Modo 2. En virtud de este rasgo social, el locus de decisión de las prioridades de investigación se desplaza de la comunidad académica al ámbito político, social y económico que contextualiza el ámbito en el que se desarrolla la academia. Así, y desde esta lógica, resulta imperativo que la investigación universitaria aborde una serie de temáticas socialmente de moda, como las vinculadas con el medioambiente, la salud o las comunicaciones, en lugar de otras menos aireadas desde un punto de vista político y social. Con lo que, frente a lo que era lógico en la práctica modernista de la universidad, que la universidad rigiera los destinos de la sociedad en la que se hallaba inserta, las exigencias postmodernas despojan a la institución universitaria de su posición rectora de privilegio y le asigna una misión subsidiaria de respuesta a los parámetros temáticos dictados por las exigencias políticas, económicas y sociales inmediatas. Nuestra postura divergente con el cambio de estatus de la institución universitaria y de los académicos integrantes de la misma, no tiene que ver con la mera pérdida de una posición 
social de la misma de primer orden a otra puramente instrumentalizada. Nuestra disconformidad con esta propuesta atañe a nuestro convencimiento de la idoneidad de que sea el grupo social más formado, la comunidad académica, la que guíe a la clase política y social por los mejores destinos humanos, en lugar de que sea la inmediatez y contingencia cambiantes de las inquietudes esporádicas de los grupos político y social, la que, carente de visión y proyecto social a largo plazo, determine la marcha de las sociedades occidentales.

Las humanidades poseen como cometido esencial la creación de cultura, valores y significados para toda la experiencia humana. A través de sus vínculos con la industria de la cultura, las humanidades configuran de manera real y fehaciente los estilos de vida, los valores y la cultura política de las sociedades occidentales. Por la relevancia social de las humanidades, resulta oportuno dedicar un espacio de análisis a la relación de la epistemología de estas ciencias y el nuevo Modo 2 de producción del conocimiento.

La relación de las humanidades con el nuevo Modo 2 de producción del conocimiento es compleja y ambivalente. Así, por un lado, podemos decir, siguiendo las apreciaciones de Gibbons et al.(1997) y desde la reflexión de las practicas sociales actualmente operantes, que en las humanidades pueden encontrarse rasgos contenidos en la producción del conocimiento Modo 2. Entre las coincidencias y similitudes de ambas formas de producción del conocimiento se encuentran los atributos de la expansión de la producción, la responsabilidad social, la transdisciplinaridad, el rol cada vez más significativo de la comercialización, y la permeabilidad de las fronteras entre los sistemas universitario y científico, por un lado, y entre la sociedad y la economía, por el otro. El impacto que actualmente los sistemas políticos, económicos y sociales ejercen sobre el sistema universitario se concreta en implicaciones puntuales como la que se cifra en que el control de calidad ya no viene determinado por la comunidad académica o comunidades concretas de expertos, sino que supone la convalidación a partir de otras fuentes externas e incluye criterios más amplios. Esto es muy visible en los imperativos que, en los últimos años, las agencias políticas gubernamentales han demandado al ámbito de las revistas científicas. Antaño las publicaciones periódicas eran gestionadas en sus distintos aspectos editoriales y de calidad por el grupo de expertos que lideraba el nacimiento y el desarrollo de una revista científica concreta. No obstante, en los últimos años se han creado agencias gubernamentales como la Fundación Española para la Ciencia y la Tecnología (FECYT) que imponen sus criterios cualitativos a los académicos que gestionan las diversas revistas científicas, con el objeto de que estas publicaciones se conformen a unos criterios dados de evaluación, profesionalización, calidad informativa, calidad de los procesos editoriales, calidad científica, difusión y visibilidad, accesibilidad e internacionaliza- 
ción. La meta de estas políticas editoriales radica en incrementar la evaluación, la profesionalización editorial y la adecuación a indicadores científicos internacionales. Adicionalmente, se han creado una serie de índices bibliométricos, como el INRECs o el RESH, que proporcionan información estadística acerca del recuento de citas bibliográficas, con el objeto de valorar la relevancia, el influjo y el impacto de las revistas científicas, de los artículos que se publican en las mismas, y de las instituciones académicas en las que se incardinan sus autores. Los modelos de referencia son los establecidos por editoriales comerciales como la Web of Science. El objeto de estas medidas radica en incrementar la visibilidad, la internacionalización y el impacto de las publicaciones periódicas, con el fin de mejorar la reputación internacional de la ciencia en un país. Las bases de datos del ISI, y en concreto la base de datos del Social Science Citation Index (SSCI) de Thomson Reuters se ha constituido en el paradigma de lo que constituye buena forma de hacer ciencia. Los equipos editoriales universitarios distan de poseer ya la tradicional autonomía de que disfrutaran en épocas pasadas, y deben dedicar sustanciales esfuerzos académicos y de gestión a conformar sus publicaciones a los criterios editoriales exigidos por las editoriales comerciales consideradas reputadas.

El intervencionismo político en el ámbito de las publicaciones periódicas evidencia, qué duda cabe, el impacto de los atributos del Modo 2 en el ámbito de las humanidades, y la participación de estas en las exigencias de aquellos. No obstante, los mismos proponentes del surgimiento del Modo 2 de producción del conocimiento cuestionan la plena participación de las humanidades en la forma de hacer ciencia del Modo 2. Las humanidades poseen un resquicio conservador y "preindustrial e incluso antiindustrial" (Gibbons et al., 1997) que provoca que, en buena parte, no participen plenamente o en su totalidad de la corriente postmoderna en la cultura y de sus derivaciones fragmentarias, relativistas, efímeras nacidas paralelamente a la actual transformación postindustrial de la economía. Algunas corrientes en el seno de las humanidades participan y estimulan el ethos conservador y tradicional en la academia, y estas ciencias han sido convocadas en momentos de fragmentación social y de cambio económico cuando se intentaban resaltar los valores tradicionales, como forma de contrarrestar la atomización postindustrial. Gibbons et al.(1997) muestran cómo fue precisamente en momentos de desregulación a gran escala de los mercados financieros y la privatización de grandes partes del estado de bienestar durante la década de los 1980 cuando se impuso con fuerza en los EE.UU. el movimiento por el alfabetismo cultural, cuando se implantó por vez primera el currículo nacional en las escuelas británicas, y cuando en la Francia socialista se produjo un regreso a los viejos valores educativos, presentados ahora como virtudes republicanas. 
Las exigencias que el Modo 2 de producción del conocimiento imponen a la institución universitaria revela hasta qué punto los paradigmas neoliberales de la responsividad y la rendición de cuentas en educación, impulsados en la era Thatcheriana en un contexto de recesión económica, pretenden gobernar la universidad actual. Hay toda una serie de cambios institucionales que han sido señalados como inminentes por los expertos. Entre estos destacan el crecimiento del trabajo transdisciplinar, la intensificación de las interacciones entre la ciencia, la tecnología y los temas sociales, la emergencia de criterios extracientíficos en la determinación de las prioridades de la investigación, el desarrollo de una ciencia participativa, la multifuncionalidad de las universidades, y el carácter híbrido del control de la calidad de la producción científica (Gibbons et al., 1997).

La embestida epistemológica postmoderna pretende asemejar la institución universitaria a todas las restantes instituciones productoras de conocimiento que han surgido de forma reciente, que realizan una diversidad de funciones, y que interactúan con otras agencias como la industria. En los actuales momentos de crisis económica, el atributo de la universidad moderna de disinterestedness (Filmer, 1997), necesario para la construcción de una ciencia coherente y con visión a largo plazo, constituye un lujo que los gobiernos no se pueden permitir. El carácter no jerárquico del Modo 2 de producción del conocimiento, concepción que se extiende a su idea social y cultural de este mismo carácter, determina lo que ha sido denominado «pluralización de la función de élite». Lo cual significa la erosión del atributo de la universidad de máxima experta epistemológica de la sociedad. Los gobiernos, atendiendo ahora a criterios económicos y políticos, crean nuevos comités burocráticos con el cometido de asignar la etiqueta de excelencia a las instituciones que cumplen con los nuevos criterios extracientíficos por ellos diseñados.

Las temáticas de estudio académico deben incorporar, para la obtención de subvenciones para la investigación, "formas híbridas» de trabajo disciplinar (Gibbons et al., 1997), y cuestiones de relevancia social y cercanas a los contextos de uso que contribuyan a resolver problemáticas del mundo real.

Las tendencias epistemológicas postmodernas plantean también exigencias a los componentes de la comunidad académica, a los cuales se pide que asuman múltiples identidades cognitivas necesarias para desarrollar un trabajo transdisciplinar. No está claro cómo un académico tiene que conjugar estos atributos propios de «analista simbólico», de formación polivalente y amplia, con la especialización que le exige el estudio profundo del ámbito disciplinar en el que desarrolla su labor cotidiana. 
La reivindicación que en este escrito realizamos del ethos y de la función moderna y tradicional de la universidad no tiene que ver con una resistencia institucional a la adaptación a los nuevos tiempos. La apuesta por el desarrollo de los atributos clásicos de la función académica se cifra en nuestra visión de que la misión de la universidad radica en la búsqueda de la verdad y en la conducción de los destinos de la sociedad, apoyada y basada en una tradición y en un patrimonio epistemológico de siglos, que posee la capacidad de vislumbrar el mejor camino de avance actual y futuro para la felicidad y la solidaridad humanas. Esta importante misión universitaria sólo la podrán ejecutar los académicos si se les permite trabajar sin atender a estrictas imposiciones y condicionantes cambiantes del mundo político y, sobre todo, económico. En tiempos de crisis económica, la universidad también debe contribuir al consejo y a la orientación gubernamental. Pero si se le exige de forma impuesta y obligada que modifique su ethos y su misión social tradicional, toda la sociedad a largo plazo sufrirá del empobrecimiento moral y social de este reduccionismo económico y epistemológico. No creemos que deba equipararse la universidad con otras instituciones creadoras de conocimiento que han surgido recientemente. $\mathrm{Ni}$ creemos que deba imponerse a la universidad la adecuación y adaptación de sus prácticas organizativas y epistemológicas acorde a las que dichas instituciones realizan. Simplemente poseen misiones y funciones diversas.

La transformación de la producción del conocimiento ha supuesto grandes cambios en el enfoque del pensamiento político. Hace dos décadas el pensamiento político respecto de la ciencia apuntaba al crecimiento de la empresa científica, delegando las decisiones clave de la ciencia en los científicos, los cuales las emprendían atendiendo a los desarrollos intrínsecos de las disciplinas. La crisis económica occidental que se inició a finales de la década de los setenta orientó un cambio sustancial del pensamiento político respecto de la ciencia. La nueva política científica apunta a la innovación industrial y la competitividad, y reivindica el rol de la ciencia en el crecimiento económico nacional e internacional.

El propio ámbito político ha experimentado cambios sustanciales: en la configuración del pensamiento político intervienen ahora expertos e industriales y las prioridades científicas se generan en el seno de foros híbridos compuestos por actores diferentes y tienen una dimensión social. La nueva política científica y de creación del conocimiento Tipo 2 se basa en las personas y en la competencia. Promueve la transdisciplinaridad y la colaboración internacional. Gestiona la interconexión entre competencia y colaboración. Estimula el crecimiento de las redes internacionales y de la permeabilidad. Tiende a afrontar el desequilibrio potencial entre volatilidad y permanencia de las instituciones. Estimula la diversidad y promueve la ex- 
perimentación y la creatividad. Concibe las especialidades como con carácter multidisciplinar y orientadas a la resolución de problemas.

Los proponentes del crecimiento de la producción del conocimiento Modo 2 especifican que la nueva política necesitará de instituciones diferentes, más ágiles, promotoras de redes de innovadores y sometidas a evaluación periódica. Coincidimos con la propuesta de Gibbons et al.(1997) en relación a la necesidad de contar con instituciones de nuevo cuño sometidas a los parámetros de la responsividad y rendición de cuentas al ámbito económico. La institución universitaria, que en los últimos años ha experimentado también las exigencias de agencias evaluadoras de su acción como la Agencia Nacional de Evaluación de la Calidad y la Acreditación (ANECA), debe contar con un margen de autonomía para desarrollar su labor educadora y su formación de «analistas simbólicos» que reivindica la sociedad del conocimiento. Es decir: personas cuya excelencia analítica de la información y del conocimiento es sólo aportada a través de una sólida y prolongada formación académica. Algunos académicos alertan del peligro de plegar de forma tan estrecha la enseñanza a las demandas profesionales. Lo cual puede derivar en una "pérdida de la energía intelectual esencial y divergente necesaria para producir formas de pensamiento y creatividad que subyacen a las aplicaciones orientadas al mercado» (King, 2004, 55).

Entre las temáticas de interés y de inquietud en el futuro destacan dos: la naturaleza y la composición de las identidades profesionales, y la contribución del conocimiento socialmente distribuido a las desigualdades mundiales, en términos del acceso y utilización de los resultados de la actividad científica. No todas las sociedades mundiales tienen infraestructura y medios para participar de los beneficios del Modo 2 de producción del conocimiento. Este déficit democrático del Modo 2 de producción del conocimiento amenaza, en plena constitución de la sociedad del conocimiento, con «transformar de nuevo la educación en un bien oligárquico» (Ball, 2006, 69).

\section{CONCLUSIÓN}

La globalización, la expansión de las nuevas tecnologías, y los cambios económicos, políticos y sociales que se han derivado de estos procesos han potenciado el debate educativo y el cuestionamiento por los académicos postmodernos de la idoneidad de los planteamientos y paradigmas tradicionales en educación. Así, actualmente es abundante la literatura que somete a revisión los fundamentos teóricos y la práctica educativa del paradigma relativo a la creación del conocimiento. Académicos como Holmes advierten, no obstante, de la coexistencia de paradigmas educativos en el ámbito de las Ciencias Sociales en el que nos hallamos insertos. 
En relación al modo de producción del conocimiento, cabe afirmar que, hoy día, resulta aún una incógnita el hecho de la extensión e implantación académica del Modo 2 de producción de conocimiento. La existencia de modelos institucionales y académicos de prácticas epistemológicas tan diversas como la Universidad de Oxford y la Universidad para la Industria británica, respectivamente, sugiere la coexistencia de ambas formas de producción de conocimiento, más que el avance gradual del Modo 1 hacia el Modo 2. En todo caso, hay algunas cuestiones de extraordinaria relevancia, relativas al estatus de las diversas ciencias, a los problemas epistemológicos abordados en la academia, y al futuro de nuestras sociedades académicas y publicaciones periódicas, que deben ser más ampliamente debatidas para evaluar el posible efecto nocivo de adoptar a ciegas el Modo 2 de creación de conocimiento en la universidad. Una de las implicaciones más relevantes, en este sentido, radica en el cambio de concepción de la propia institución universitaria y de su misión respecto de la sociedad que se deriva de la adopción del Modo 2 de creación del conocimiento. La universidad no puede abdicar del rol social que dio origen y sentido a su creación: enseñar a los estudiantes a pensar críticamente respecto de la sociedad en la que se inserta. 


\section{REFERENCIAS BIBLIOGRÁFICAS}

AA.VV. (2002). Towards the End of Education Systems? Europe in a world perspective. Abstracts Booklet CESE Conference 2002. London: Institute of Education: University of London.

Cárceles Laborde, C. (2006). Las tendencias curriculares en las instituciones educativas contemporáneas, en Vergara Ciordia, J. (Coord.). Historia del currículo. Madrid: UNED.

Cowen, R. (1996). Performativity, Postmodernity and the University, Comparative Education, 32, 245-258.

Dale, R. (2007). Specifying Globalization Effects on National Policy, en Lingard, B. y Ozga, J., Education Policy and Politics. London: Routledge.

DfEE (1998). The Learning Age: a renaissance for a new Britain. Sheffield: Department for Education and Employment.

Drori, G. S., Meyer, J.W., Ramirez, F.O. y Schofer, E. (2006). La ciencia en la política mundial moderna. Barcelona: Edit. Pomares.

Field, J. (2000). Lifelong Learning and the new Educational Order. Stoke on Trent: Trentham Books.

Filmer, P. (1997). Disinterestedness and the Modern University, in Smith, A. and Webster, F. (eds.) The Postmodern University? Contested Visions of Higher Education in Society. Buckingham: SRHE and Open University Press.

García Garrido, J. L. (2000). La sociedad educadora. Madrid: Fundación Independiente.

García Garrido, J. L. y García Ruiz, M. J. (2005). Temas Candentes de la Educación en el siglo XXI. Madrid: Ediasa.
García Ruiz, M. J. (2010). Implications of the new social characteristics of knowledge production, in Mattheou, D.: Changing Educational Landscape: Educational Practices, Schooling Systems and Higher Education - A comparative perspective. Amsterdam: Springer.

Gibbons, M. et al. (1997). The New Production of Knowledge. The Dynamics of Science and Research in Contemporary Societies. London: Sage Publications.

Gibbons, M. (2004). Globalization, Innovation and Socially Robust Knowledge, in King, R. The University in the Global Age. Basingstoke: Palgrave Macmillan.

Holmes, B. (1986). Paradigm Shifts in Comparative Education, en Altbach, P. G. y Kelly, G. P. New Approaches to Comparative Education. Chicago and London: The University of Chicago Press.

Jacob, M. and Hellström, T. (eds.) (2000). The Future of Knowledge Production in the Academy. Buckingham: SRHE and Open University Press.

Jarvis, P. (2007). Globalisation, Lifelong Learning and the Learning Society. Sociological Perspectives. London: Routledge.

Ker, I. (1999). Newman's Idea of a University. A Guide for the Contemporary University?, in Smith, D., and Langslow, A.K. (eds.) The Idea of a University. London: Jessica Kingsley Publishers.

Lowe, R. (2007). The Death of Progressive Education. How teachers lost control of the classroom. London: Routledge. 
McKinsey Report (2007). How the world s best-performing school systems come out on top. McKinsey \& Company. [en línea] Disponible en: http://www.teacherqualitytoolbox.eu

NAGCELL (1997). Learning for the Twenty-First Century. First report of the National Advisory Group for Continuing Education and Lifelong Learning (Fryer Committee). London: DfES.

Ortega y Gasset, J. (1982). Misión de la universidad y otros ensayos sobre educación y pedagogía. Madrid: Alianza Editorial.

Pereyra, M. A. (Ed.)(2008). Changing Knowledge and Education. Communities, Mobilities and New Policies in Global Societies. Frankfurt am Main: Peter Lang.

Popkewitz, T. S., Franklin, B. M. y Pereyra, M. A. (Comp.) (2003). Historia cultural y educación. Ensayos críticos sobre conocimiento y escolarización. Barcelona: Edic. Pomares.

Sanz Fernández, F. (2002). Crítica a los sistemas de enseñanza, en Tiana Ferrer, A., Ossenbach Sauter, G. y Sanz Fernández, F. (Coord.) Historia de la Educación (Edad Contemporánea). Madrid: UNED.

Sanz Fernández, F. (2006). El aprendizaje fuera de la escuela. Tradición del pasado y desafío para el futuro. Madrid: Ediasa.

Spring, J. (2006). Pedagogies of Globalization. The rise of the Educational security state. London: Lawrence Erlbaum Associates Publishers.

Torres, R. M. (2003). Aprendizaje a lo largo de toda la vida: un nuevo momento y una nueva oportunidad para el aprendizaje y la educación básica de adultos en el Sur. ASDI (Estocolmo), IIZ-DVV (Bonn) y IIEP-UNESCO (Paris).

Watson, K. (ed.) (2001). Doing Comparative Education Research. Issues and Problems. Oxford: Symposium Books. 


\section{PALABRAS CLAVE}

Globalización, Universidad, postmodernismo, epistemología.

\section{KEYWORDS}

Globalization, University, postmodernism, epistemology.

\section{PERFIL ACADÉMICO Y PROFESIONAL DEL AUTOR}

María José García Ruiz, Profesora Titular de Escuela Universitaria en la Facultad de Educación de la UNED. Es miembro de la Junta Directiva de la Sociedad Española de Educación Comparada (SEEC), Secretaria de la SEEC, y pertenece a la Comparative Education Society of Europe. Es Subdirectora de la Revista Española de Educación Comparada. Sus líneas de investigación se focalizan en la enseñanza superior y las políticas educativas exteriores.

Dirección del autor: María José García Ruiz

Facultad de Educación

Dpto. de Historia de la Educación y Educación Comparada

Universidad Nacional de Educación a Distancia (UNED)

C/. Senda del Rey $n^{\circ} 7$

28040 Madrid

E-mail: mjgarcia@edu.uned.es

Fecha Recepción del Artículo: 07. Abril. 2010

Fecha Revisión del Artículo: 28. Mayo. 2010

Fecha Aceptación del Artículo: 29. Septiembre. 2010

Fecha de Revisión para publicación: 16. Mayo. 2011 
